\title{
Soft skills turned into hard facts: nucleosome remodelling at developmental switches
}

\author{
M Chioda and PB Becker \\ Adolf-Butenandt-Institute and Center for Integrated Protein Science (CiPSM), Ludwig-Maximilians-University, Munich, Germany
}

\begin{abstract}
Nucleosome remodelling factors are regulators of DNA accessibility in chromatin and lubricators of all major functions of eukaryotic genomes. Their action is transient and reversible, yet can be decisive for irreversible cell-fate decisions during development. In addition to the well-known local actions of
\end{abstract}

nucleosome remodelling factors during transcription initiation, more global and fundamental roles for remodelling complexes in shaping the epigenome during development are emerging. Heredity (2010) 105, 71-79; doi:10.1038/hdy.2010.34; published online 7 April 2010

Keywords: chromatin; histone; ATPase; nucleosome remodelling

\section{Introduction}

Embryonic development manifests as a series of changes in cellular programmes that mark the transitions from the totipotent zygote to increasingly more differentiated cells with specialized morphology and function. These programmes are best characterized by the distinct patterns of gene expression reflecting the selective usage of the genetic information. The initiation of differential gene expression is interwoven with changes in chromatin features; its maintenance is based on the existence of stable, heritable chromatin structure. Understanding developmental switches in gene expression requires detailed knowledge about the local and global changes in chromatin organization and their functional consequences.

Chromatin affects gene expression at several levels of structural organisation: through the placement of single nucleosomes, the folding of the nucleosomal fibre, the assembly of higher-order structure by interacting proteins and the formation of nuclear compartments characterized by enrichment of specific regulators. The regulatory 'add-on' to the necessity of packaging and protecting eukaryotic genomes from damage is illustrated by two major principles. First, the accessibility of DNA - genes and regulatory elements - can be tuned through differential packaging. Second, specific loci, areas or chromosomal domains may be earmarked as substrates for those enzymes that translate structure into function (Allis et al., 2007). Post-translational modifications of histones, the constituents of nucleosomes, very obviously serve both principles (Bonisch et al., 2008; Suganuma and Workman, 2008; Wood et al., 2009). Less obvious is the contribution of the so-called adenosine-5'triphosphate (ATP)-dependent nucleosome remodelling enzymes that will be the focus of this review.

Correspondence: Professor PB Becker, Adolf-Butenandt-Institute, Molecular Biology, Ludwig Maximilians University, Schillerstrasse 44, Munich D-80336, Germany.

E-mail: pbecker@med.uni-muenchen.de

Received 12 November 2009; revised 25 February 2010; accepted 25

February 2010; published online 7 April 2010

\section{ATP-dependent nucleosome remodelling}

Nucleosome remodelling factors are enzymes that are able to disrupt the tight ionic association of DNA with histones in the canonical nucleosomes or nucleosome assembly intermediates. Key to their action is an ATPase 'motor' subunit that belongs to the large SNF2 family of helicase-related proteins, many of which are able to translocate DNA. The simultaneous movement of DNA and binding to the histones leads to displacement of DNA segments relative to the histone surface. The conformational changes in the enzyme necessary for this action are supported by binding and hydrolysis of ATP (Clapier and Cairns, 2009). Depending on the circumstances, the action of these 'remodelling factors' may lead to the disassembly of entire nucleosome (their eviction), or the removal of the 'outer' $\mathrm{H} 2 \mathrm{~A}-\mathrm{H} 2 \mathrm{~B}$ histone dimer, effectively generating stretches of accessible DNA. However, remodelling factors can also catalyse the reverse reactions, the assembly of nucleosomes from histones bound to chaperones and the completion of nucleosomes from which the $\mathrm{H} 2 \mathrm{~A}-\mathrm{H} 2 \mathrm{~B}$ dimers have been removed, for example, by incorporation of $\mathrm{H} 2 \mathrm{~A}$ variants (Clapier and Cairns, 2009). Nucleosome remodelling may also lead to the repositioning of intact histone octamers (that is, nucleosomes) on DNA. The movement of nucleosomes may generate access for DNA-binding regulators, or, conversely, close gaps in the nucleosomal fibre by equal spreading of nucleosomes (Becker, 2002). The regularity of spacing of nucleosomes and the internucleosomal distances affect the folding of the nucleosomal fibre (Routh et al., 2008). Placement of nucleosomes can have a profound effect on the higher-order chromatin structure (Varga-Weisz and Becker, 2006).

ATP-dependent chromatin remodellers are multisubunit complexes conserved through evolution. Their 'motor' subunit is an ATPase of one of the 24 subfamilies of the SNF2 family, which are defined by similarity in sequence and domain organization (Flaus et al., 2006). The best-studied ATPases in terms of nucleosome remodelling belong to one of four subfamilies 
represented by the ATPases SWI2/SNF2, ISWI, CHD/ Mi2 and Ino80/SWR1. Simple remodelling factors may just consist of the ATPase alone, or one additional subunit. The most complicated remodelling machines have more than 15 subunits (Clapier and Cairns, 2009) that serve to regulate their activity, to target them to sites of action and to integrate the remodelling activity into physiological processes. Nucleosome remodelling factors are facilitators that endow chromatin with plasticity and flexibility to respond to environmental and developmental cues. Because of the dynamics of the transitions of histone-DNA interactions, their action is difficult to observe in vivo. The transient unfolding and local movement of nucleosomes leave no trace. The importance of nucleosome remodelling for the developmental processes discussed in this study can usually be deduced only from loss-of-function phenotypes.

We will review the role of the nucleosome remodelling factors during development or stem cell differentiation by highlighting recent examples in which the dynamic and reversible remodelling action contributes to taking irreversible developmental decisions. Earlier findings have been summarized elsewhere (de la Serna et al., 2006; Kwon and Wagner, 2007; Keenen and de la Serna, 2009; Yoo and Crabtree, 2009). We first consider how local, targeted nucleosome remodelling directly affects the transcription of genes coding for developmental regulators and illustrate the factor complexity that underlies the selective remodelling. In the second part we introduce the emerging concept that remodelling machines are critical for the global structural 'stratification' of the epigenome in the process of diversification of chromatin organization as stem cells differentiate. In this context, non-nucleosomal targets for remodelling machines (such as the linker histone H1) and effects on higher-order chromatin structure are considered, which leads to the concept of 'chromatin remodelling factors' with broader substrate selectivity.

\section{Local complexity of nucleosome remodelling at promoters}

During embryonic development, cell type-specific master regulators induce cell differentiation by installing specific transcription programmes. This process is particularly well characterized in Drosophila melanogaster development, in which a cascade of gene expression programmes of increasing subtlety specifies the body plan of the organism and all cell identities. ATPdependent nucleosome remodelling is an integral part of transcription initiation at most complex eukaryotic promoters, in which the remodelling machines are usually recruited to selected targets by sequence-specific transcription factors (Becker and Horz, 2002). The intricate developmental transcription networks are no exception to this common principle. Faithful differentiation, however, relies as much on transcriptional repression as it does on activation. Differentiation genes have to be kept silent in stem cells and pluripotent precursors and similarly lineage-specific programmes involve repression in cells with different commitment. Nucleosome remodelling is involved in both activation and silencing.

The regulation of the expression of wingless during development may serve to illustrate the notion that chromatin remodelling factors may have opposite effects on transcription (Liu et al., 2008; Song et al., 2009). The evolutionary conserved wingless (Wg)/Wnt signalling pathway controls many developmental processes, including cell differentiation and stem cell maintenance in adult tissues (Logan and Nusse, 2004; Cadigan and Liu, 2006; Cadigan, 2008; Nusse et al., 2008). Expression of wingless leads to stabilization of $\beta$-catenin/armadillo, which enter the nucleus where it associates with several co-activators to bind to Wnt/Wg target promoters to induce transcription. Recently, it has become clear that nucleosome remodelling factors of the ISWI type are among the co-regulators of Wg-dependent transcription. The ATPase ISWI is shared by several chromatin remodelling factors, among them the well-studied factors NURF and CHRAC/ACF (Tsukiyama et al., 1995; Ito et al., 1997; Varga-Weisz et al., 1997). Although NURF and CHRAC/ACF-due to their shared ATPase subunit ISWI-exert an effect predominantly through nucleosome sliding, the outcome of the nucleosome mobilization is different. NURF is able to disrupt regular nucleosomal fibres in vitro, whereas CHRAC/ACF promotes the assembly of regularly spaced chromatin fibres (Langst and Becker, 2004). NURF, but not CHRAC/ACF, has been implicated in transcriptional activation before (Badenhorst et al., 2002; Fyodorov et al., 2004). For example, a genome-wide expression analysis of mutants lacking the NURF-specific subunit NURF301 revealed a large set of ecdysone-responsive targets among several hundred NURF-regulated genes (Badenhorst et al., 2005). In the absence of NURF, larvae fail to proceed through metamorphosis. Purified NURF binds the ecdysone receptor in an ecdysone-dependent manner, suggesting that is may be targeted by the receptor to target promoters to affect transcription. NURF has now been found to promote transcription of $\mathrm{Wg}$ target genes by being targeted to their promoters through the direct interaction with Armadillo (Song et al., 2009). Upon Nurf301 depletion in flies some, but not all, Wg target genes were no longer expressed in larvae. Interestingly, global expression analysis in Drosophila Kc cells recently suggested that the ISWI-containing CHRAC/ACF remodeller may contribute to maintaining a basal repression of $\mathrm{Wg}$ target genes in the absence of Wg ligand (Liu et al., 2008). Wingless stimulation reduced the binding of ACF1 to $\mathrm{Wg}$ target genes, leading to the hypothesis of an antagonism between two different nucleosome remodelling factors with a shared ATPase subunit.

The different behaviour of these two ISWI-containing factors on the same signalling pathway sheds lights on the importance of the regulated targeting of these two complexes. One principle that could favour the recruitment of one complex rather than the other might be a tight developmental expression of the subunits forming CHRAC/ACF or NURF. Indeed, in cleavage nuclei and early gastrulation when the $\mathrm{Wg} / \mathrm{Wnt}$ signalling pathway is not yet fully activated, ACF1, the defining subunit of CHRAC/ACF, is particularly abundant (Ito et al., 1999; Chioda et al., 2010). ACF1 expression is greatly reduced as development proceeds and NURF becomes the main ISWI complex at later developmental stages when transcription of $\mathrm{Wg}$ target genes is activated. The importance of regulating the expression of subunits defining different chromatin remodelling factors has also been revealed by the finding that at least three different 
splice variants of the large Nurf301 subunit exist in vivo (Kwon et al., 2009). One of them lacks the C-terminal plant homeodomain finger (PHD) and a bromodomain. These C-terminal domains are apparently largely dispensable for the above-mentioned NURF targets, but are crucial for spermatogenesis. In the absence of this targeting module, spermatocytes arrest differentiation and do not express a subset of spermatid differentiation genes.

Although the in vitro activities of NURF and CHRAC/ ACF support a dichotomy of function-activating and repressing - the situation is likely much more complicated, as NURF can apparently also function as a corepressor of transcription. Badenhorst and colleagues showed that the transcription repressor Ken may recruit NURF to target genes of the JAK/STAT (janus kinase/ signal transducers and activator of transcription) pathway and that these target genes are precociously activated in the absence of NURF (Kwon et al., 2008).

There are also examples of antagonistic regulatory roles for distinct chromatin remodelling complexes on transcription of developmental genes in other model systems. For example, in the mouse bone marrow, the development of B cells from common progenitors is tightly regulated through a transcriptional network, orchestrated by the 'master' regulator EBF (early B-cell factor). EBF is necessary for inducing immunoglobulin gene rearrangement and B-cell differentiation; and without it B-cell differentiation is arrested at the level of early progenitors. EBF-dependent activation of genes required for B-cell differentiation involves promoter opening and DNA demethylation. This also involves the action of at least two nucleosome remodelling complexes with antagonizing functions (Gao et al., 2009). The large remodelling complex SWI/SNF is recruited to the EBF promoter target $m b-1$, in which it catalyses nucleosome repositioning in preparation for activation by Pax5. The precocious activation of the promoter is prevented by the repressive chromatin remodeller NuRD, powered by the Mi2b ATPase, which is suggested to function as a 'gatekeeper' that prevents differentiation under inappropriate circumstances (Gao et al., 2009). NuRD complexes are particularly widespread repressors that combine ATP-dependent nucleosome remodelling by a CHD3/4-type ATPase with histone deacetylases in one complex. The versatility of its use is reflected by the heterogeneity of the complex because of the existence of cell type-specific variants of the individual subunits (see Bowen et al., 2004, and references therein). A particularly nice example of the programmatic action of $\mathrm{NuRD}$ complexes comes from the Xenopus model, in which Rupp and colleagues showed that the boundary between mesoderm and neuroectoderm along the animal-vegetal axis at gastrulation are controlled by the levels of Chd4/ Mi-2 $\beta$ (Linder et al., 2007).

The action of remodelling factors is not only limited to promoter regions, but may also affect transcription at the level of elongation. This may be the case for Drosophila Kismet (Srinivasan et al., 2008), a chromatin remodelling ATPase belonging to the CHD subfamily of the SWI2/ SNF2 ATPases (three apparent mammalian orthologues are called CHD7 and CHD8). Kismet is one of the chromatin remodelling factors that genetically interact with the regulatory factors of the Polycomb and trithorax groups. These groups of proteins are necessary to propagate the activity patterns of Hox genes, which are established during early embryonic development. Correct regulation by the right balance of negative (Polycomb group proteins) and positive regulators (Trithorax group proteins) is crucial as inappropriate expression of Hox genes leads to severe developmental defects and homeotic transformations. This is the case when Kismet is depleted. It has recently been suggested that Kismet may affect transcriptional elongation. Kismet was found necessary for recruitment of ASH-1 (Absent, Small, or Homeotic 1) and TRX (Trithorax) to transcriptionally active genes, with which it exerts an effect in concert to prevent spreading of the repressive histone mark H3K27me3 mediated by Polycomb complexes (Srinivasan et al., 2008). Although the mechanistic details of this phenomenon are not clear, the researchers discuss the possibility that Kismet may limit normal H3K27me3 levels by promoting the exchange of canonical, K27methylated $\mathrm{H} 3$ with the variant histone $\mathrm{H} 3.3$ along the transcribed regions. H3.3 is indeed particularly abundant at sites of active transcription, and enrichment of H3.3 has been observed at Polycomb and TRX-binding sites near the hox genes (Mito et al., 2007). As Kismet has not been biochemically characterized yet, it remains unclear whether the remodeller is directly involved in histone exchange reactions of this type or whether the effect is more indirect because of stimulation of elongation. The mammalian orthologue CHD8 has nucleosome mobilization activity in vitro and was recently found associated with the elongation form of RNA polymerase II, supporting a role in elongation. Prominent target genes code for $\beta$-catenin, cyclin E2 and thymidylate synthase, which explains the fact that depletion of CHD8 leads to proliferation defects (Rodriguez-Paredes et al., 2009). Human CHD7 is mutated in CHARGE syndrome patients who suffer from a number of developmental defects. Wysocka and colleagues recently showed that CHD7 activity is crucial for formation of the multipotent migratory neural crest cells that are the precursors of a variety of cells that are lacking in CHARGE patients (Thompson et al., 2008; Bajpai et al., 2010). Interestingly, these researchers found CHD7 physically associated and functionally synergizing with the PBAF complex (see below), suggesting a complex cooperation of different ATP-dependent remodellers during cell programming (Bajpai et al., 2010).

Verrijzer and colleagues recently suggested another mechanistic explanation for the antagonism between a nucleosome remodelling factor and the repressive Polycomb system (Kia et al., 2008). Hypothetically, the SWI2/SNF2-type Brahma complex in Drosophila is directly involved in the removal of repressive Polycomb complexes from silencers. The concept that nucleosome remodelling factors are not only suited to disrupt histone-DNA interactions, but may also affect the DNA binding of other chromatin proteins, is novel and will be recurring later in this summary.

The examples mentioned above already illustrated some recurring principles that assure the selective action of remodelling factors. Remodelling factors may be targeted to promoters and other sites by direct interaction with DNA-bound activators or repressors. This interaction usually does not directly involve the ATPase subunit, but one of the associated subunits that define the specific context for action. The availability and properties of these subunits (in the case of splice 
variants, see above) may be under tight cell type-specific and developmental control. A nice case for cell typespecific targeting during differentiation is the case of the above-mentioned Brahma complex. In Drosophila, two variants of this complex exist: BAP and PBAP share seven subunits and both complexes counteract Polycomb silencing. However, the two complexes are distinguished by individual 'signature subunits'. A module consisting of Polybromo, BAP170 and SAYP specifies PBAP, whereas Osa defines BAP. The signature subunits are critical for selective targeting and for the faithful functioning of the corresponding 'core' complexes in vivo (Chalkley et al., 2008; Terriente et al., 2008). SWI/SNF-type complexes are evolutionarily conserved regulators, from yeast to man. The mammalian homologues of the Drosophila BAP and PBAP complexes are called 'BAF' complexes in mice and, as in flies, a variety of cell-type-specific forms exist. wewBAF complexes are necessary for the self-renewal and pluripotency of mouse embryonic stem (ES) cells but not for the proliferation of other cells. ES cells express distinct complexes (esBAF) defined by the presence of the SWI2-type ATPase Brg (Brahma-related gene), BAF155 and BAF60A, and the absence of Brm (Brahma), BAF170 and BAF60C. Crabtree and colleagues showed that this specialized subunit composition is required for ES cell maintenance and pluripotency and that esBAF complexes interact directly with key regulators of pluripotency, suggesting a molecular explanation for their important role in maintaining pluripotency (Ho et al., 2009a, b). In addition to esBAF, variant complexes with different signature subunits are dedicated to channelling the neurogenic lineage, such as npBAF, which is specific to neural progenitors, or $\mathrm{nBAF}$, which is found in post-mitotic neurons (see Yoo and Crabtree, 2009 and references therein)

\section{Global roles: the diversification of the epigenome}

In addition to the local, gene-specific contributions of remodelling factors that manifest directly in transcription programmes conferring cell identity, there is accumulating evidence for more global, genome-wide roles for these enzymes. According to the emerging concept, the increasing definition of cell type-specific transcription profiles is accompanied-or indeed channelled-by a progressive diversification of the 'epigenome', that is, the global arrangement of chromatin modifications and factor binding. The chromatin of differentiated cells is thought to be organized by a relatively stable chromatin fibre with a full complement of linker histones. Active and inactive chromatin (such as constitutive or facultative heterochromatin or just repressed euchromatin) can be clearly distinguished by their histone modification patterns and by the association of the enzymes establishing these marks. These histone modifications are recognized by interacting proteins that define-through unknown mechanisms-the higher-order chromatin organization.

Pluripotent cells differ from their differentiated offspring by many chromatin features, including histone and DNA modification, nucleosome density and dynamics, heterochromatin organization and chromosome arrangements in interphase nuclei (Meshorer and Misteli, 2006; Meshorer et al., 2006; Giadrossi et al., 2007). ES cells seem to be richer in less compact chromatin and lack the condensed heterochromatin foci that characterize the differentiated cells (Arney and Fisher, 2004; Meshorer and Misteli, 2006). Chromatin in stem cells lacks irreversible chromatin structures. Opposing histone modifications (known to characterize either the repressed or active states) do not segregate cleanly, but rather colocalize at sites to define a novel, 'bivalent' plastic-state (Azuara et al., 2006; Bernstein et al., 2006). ES cells also have lower amounts of the linker histone H1 when compared with differentiated cells (Fan et al., 2005; Woodcock et al., 2006). H1 stabilizes the folding of the nucleosomal fibre into higher-order structures. A reduced stoichiometry of the linker histone may thus correlate with a partial decondensation of chromatin.

The pluripotent state is characterized by 'hyperdynamic chromatin', in which core histones, linker histones and heterochromatin protein 1 (HP1) are more loosely associated with chromatin. Meshorer and Misteli (2006) suggest that hyperdynamic chromatin may contribute to the maintenance of pluripotency and to facilitate the timely formation of higher-order chromatin during differentiation (Meshorer et al., 2006).

In the following section we will concentrate on those global changes in chromatin structure during development that are likely to be mediated by ATP-depending nucleosome remodelling machines: the exchange of histone variants, the homeostasis of the association of linker histones and the integrity and regularity of the nucleosome fibre. In light of recent findings, the discussion of the incorporation of histone variants and linker histones will be largely discussed in the context of Drosophila embryogenesis and spermatogenesis. The shaping of the genome is important not only during embryonic development, but also in stem cells, which need to maintain the ability of self-renewal combined with all the potential of giving rise to different cell types. The dynamic transitions during differentiation of stem cells will be followed in the vertebrate system.

\section{Variant chromatin organized by remodelling factors}

The set of histones that constitute chromatin during earliest development are mainly the somatic replicationdependent isoforms. As differentiation proceeds, celltype specific histone variants are expressed and incorporated into chromatin, conferring defined properties to the chromatin substrate (Izzo et al., 2008; Godde and Ura, 2009; Orsi et al., 2009; Svotelis et al., 2009). The incorporation of histone variants, at least the replication independent type, is frequently achieved by cooperation between an ATP-dependent remodelling enzyme and dedicated histone chaperones (Kusch and Workman, 2007; Altaf et al., 2009). In Drosophila, early embryonic chromatin does not contain the histone $\mathrm{H} 2 \mathrm{~A}$ variant, $\mathrm{H} 2 \mathrm{Av}$, which becomes visible only from nuclear division 8 onwards, just before cellularization (M Chioda, unpublished data). The deposition of $\mathrm{H} 2 \mathrm{Av}$ is crucial for the establishment of heterochromatic structures, as larvae lacking this variant do not show a normal distribution of heterochromatic markers such as 
H3K9Me2 or heterochromatin protein 1a (Swaminathan et al., 2005). H2Av incorporation requires the chromatin remodelling factor RSF (remodelling and spacing factor), a nucleosome assembly factor powered by the ATPase ISWI (Hanai et al., 2008). RSF depletion affects the amount of $\mathrm{H} 2 \mathrm{Av}$ in facultative heterochromatin, whereas the contribution to constitutive heterochromatin of the chromocenters seems to be unaffected. Interestingly, RSF genetically and physically interacts with the Tip60 complex in flies, another remodelling complex containing the ATPase Domino, for which a nucleosome remodelling activity has been suggested but not yet shown (Hanai et al., 2008). The diversification of the epigenome into eu- and heterochromatin characteristic of differentiated cells is, therefore, brought about by at least three different remodelling machineries (see below).

A further example of the developmental role of a nucleosome remodelling factor in histone exchange is the incorporation of the histone H3.3 variant into the chromatin of the paternal nucleus of the Drosophila zygote by the single-subunit ATPase CHD1 (Konev et al., 2007). In CHD1 mutants the paternal pronucleus fails to specifically incorporate $\mathrm{H} 3.3$ and the resulting deranged chromatin prevents proper fusion with the maternal pronucleus and subsequent nuclear cleavage. H3.3containing nucleosomes are assembled by cooperation of CHD1 with the histone chaperone HirA. The formation of H3.3-containing nucleosomes rather than 'canonical' ones may be explained by the fact that nucleosome assembly must occur in the absence of DNA replication in this case. H3.1 is assembled during replication, whereas H3.3 is used in a variety of circumstances, in which nucleosomes form independently of replication (Groth et al., 2007). Although the zygote is loaded with maternally deposited remodelling factors, none of them is able to compensate for the loss of CHD1, which illustrates the selectivity of action. However, this specificity is limited to the earliest time in development, as individuals surviving these crucial phases partially rescue the incorporation of $\mathrm{H} 3.3$ into chromatin (Konev et al., 2007). H3.3 assembly may thus be catalysed by different factors during development.

CHD-type ATPases seem to be particularly suited to deal with the histone variant H3.3, as observed during chromatin assembly in the male pronucleus (CHD1) or hypothesized for incorporation of H3.3 during transcription (Kismet, see above). In contrast, CHD1 is unable to deal with the linker histone H1 (see below). The exchange of histone H2A variants is a speciality of those remodelling factors that contain a typical 'split' ATPase domain (the Swr1- type; Kusch and Workman, 2007; Altaf et al., 2009). Nucleosome remodelling factors are evidently specialized to deal with particular histone substrates.

\section{Linker histones: links between nucleosomes chromatin fibre folding}

Various circumstantial evidences suggest that nucleosome remodelling factors may not only work on assemblies with core histones and DNA, but may have other substrates as well. Recently, the linker histone H1 emerged as a putative novel substrate of remodelling factors, a particularly important substrate, as its presence has profound implications for the folding of the nucleosome fibre (Woodcock et al., 2006). Virtually all higher eukaryotes have a maternal version of the linker histone $\mathrm{H} 1$, which differs in its biochemical properties from the embryonic or somatic variants (Dimitrov et al., 1993). Pluripotent cells may have a reduced concentration of linker histones, which correlates with the flexible state of genome organization. Terminally differentiated tissues commonly contain distinct $\mathrm{H} 1$ subtypes that confer a tighter chromatin compaction (Izzo et al., 2008). Nucleosome remodelling factors differ in their ability to remodel nucleosomes in the presence of the linker histone. Those machineries, such as the ISWI-containing ACF complex (Maier et al., 2008b), that are able to move nucleosomes together with associated $\mathrm{H} 1$ may be better called 'chromatin remodelling factors' (reviewed in Maier et al., 2008a). In this study we discuss how chromatin remodelling factors can affect the incorporation of linker histones during development, again using the Drosophila paradigm as an example.

During the earliest time of development in Drosophila, cleavage chromatin does not contain any linker histone, but an abundant high-mobility group protein called HMG-D (Ner and Travers, 1994). HMG-D has been considered a 'DNA chaperone' that may assist nucleosome formation during the most rapid replication cycles in preblastoderm embryos (Ner et al., 2001). During embryogenesis HMG-D is gradually replaced by H1 (Ner and Travers, 1994); its incorporation into chromatin parallels zygotic transcriptional activation. The maturation of chromatin that is initiated by $\mathrm{H} 1$ incorporation is accompanied by a significant chromatin compaction and an approximately 10 -fold reduction of nuclear size (Ner and Travers, 1994). Tamkun and colleagues observed that in absence of the remodelling ATPase ISWI, H1 was no longer properly incorporated into the polytenic X-chromosome of male Drosophila larval salivary glands. This chromosome seems somewhat decondensed because of the enrichment of acetylation of histone $\mathrm{H} 4$ at lysine 16, a modification that correlates with the twofold enhanced activity state, which is characteristic of dosage compensation (Deuring et al., 2000; Corona and Tamkun, 2004; Corona et al., 2007). Apparently, this chromosome is sensitized to reveal the relationship between ISWI and histone $\mathrm{H} 1$ incorporation. Knockdown of $\mathrm{H} 1$ expression in flies using an RNA interference strategy led to similar phenotypes of chromatin decompaction in salivary gland nuclei (Siriaco et al., 2009). A comparison of the chromosome organisation upon $\mathrm{H} 1$ knockdown or mutation of ISWI suggests that an ISWI-containing remodelling factor, presumably NURF (Badenhorst et al., 2002), is responsible for the steady-state homeostasis of $\mathrm{H} 1$ association and hence for the higher-order organization of chromosomes (Siriaco et al., 2009). Remodelling complexes containing ISWI are known to be particularly potent in the assembly and remodelling of H1-containing chromatin in vitro (Lusser et al., 2005; Maier et al., 2008b). Whether, and if so under which circumstances, nucleosome remodellers are directly involved in assembling $\mathrm{H} 1$ in vivo is an unsolved issue.

The fact that CHD1 - in comparison with ISWI-was unable to deal with H1-containing chromatin lends further support to the concept of substrate selectivity and functional specification of remodelling factors 
(Lusser et al., 2005; Maier et al., 2008b). We imagine that the local action of remodelling factors may lead to chromatin diversification (incorporation of histone variants and linker histones), but that the resulting distinct forms of chromatin are in turn selective substrates for some 'downstream' remodelling factors, but resistant to the action of others.

\section{Nucleosome remodelling and chromosome organization}

Since, the limited access to DNA sequences packaged in chromatin posed a fundamental problem for those contemplating the mechanisms of gene expression in the context of chromatin, early work naturally emphasized the potential of nucleosome remodelling for chromatin opening. More recently, it is appreciated that nucleosome remodelling is neutral with respect to the structural transitions it brings about; nucleosome can be moved to uncover a stretch of DNA, or to close a gap in the regular succession of nucleosomes that may pose a risk for chromosome breakage. Indeed, some remodelling factors are more dedicated to the repair of chromatin as opposed to its destruction. For example, ACF1-containing nucleosome remodelling factors seem to be mainly involved in the assembly of fibres with regular nucleosome spacing (Fyodorov et al., 2004; Chioda et al., 2010). Combined with the role of ISWI-containing remodellers that promote the incorporation of linker histone H1 (see above), they assure the integrity of the nucleosome array and its folding into the next level of organization. Depletion of the diagnostic large subunit of the CHRAC/ACF remodellers, ACF1, leads to sloppy chromatin that is defective in many respects, such as defects in heterochromatin assembly (Fyodorov et al., 2004; Chioda et al., 2010). It seems that the faithful assembly of higher-order chromatin, which is mainly defined by interacting non-histone proteins, relies on a proper 'infrastructure', that is, proper nucleosomal fibre.

The ACF1-containing nucleosome remodelling factors CHRAC and ACF are particularly abundant in early embryogenesis (Fyodorov et al., 2004; Chioda et al., 2010). They may, therefore, be particularly devoted to assist the step-wise assembly of chromatin structures with increasing complexity and organization. Chromatin of undifferentiated cells is characterized by a reduced nucleosome density and a lower amount of linker histones, which renders it less compact. In addition, it lacks those histone modifications that serve as platform for the assembly of facultative and constitutive heterochromatin. Cell typespecific gene expression programmes, however, rely on regional diversification of the epigenome: its differentiation into domains of open or compact, flexible or rigid structures. The 'stratification' of the epigenome, that is, the stepwise diversification and structural complexity of chromatin that characterizes the development from pluripotent to post-mitotic, differentiated cells, may thus be majorly orchestrated by remodelling factors.

\section{On the tip of the balance: the case of the stem cell}

The arguments raised above do not fully appreciate the fact that ES cells need to fulfil two seemingly opposing demands. Their ability to initiate differentiation along any of the three germ layers requires plasticity of their epigenome. On the other hand, their ability to self-renew for a significant length of time requires a tight control that resists the temptations to differentiate. Epigenetic factors, particularly the Polycomb complexes, are known to be crucial to the maintenance of stemness (Pietersen and van Lohuizen, 2008). However, the roles for ATPdependent remodelling machines in the decision between maintenance or exit from the pluripotent state have also recently entered the discussion (Clapier and Cairns, 2009; Yoo and Crabtree, 2009). In line with their role as gene-specific co-repressors, NuRD complexes have been implicated in the repression of differentiation programmes and maintenance of pluripotency (Kaji et al., 2006; Yoshida et al., 2008).

In efforts to identify further chromatin modifiers responsible for maintaining stem cell identity, two groups recently screened for factors, which were enriched in ES cells (Gaspar-Maia et al., 2009) or that could alter ES morphology (Fazzio et al., 2008). Both studies identified important, although clearly distinct roles, for chromatin remodelling factors in maintaining stem cell identity by regulating the organization of chromatin. Gaspar-Maia et al. (2009) identified CHD1 as responsible for maintaining the fluid chromatin structure typical of ES cells. Ablation of CHD1 induced differentiation of ES cell towards the neural lineage and correlated with increase in heterochromatic markers. Surprisingly, despite of this global change in chromatin structure, only a minority of genes were affected by CHD1 depletion. Depletion of CHD1 did not lead to full differentiation, as some ES markers, such as Oct- 4 or SSEA1, and an ES-like colony morphology were still retained. In the absence of further information, one can only speculate about the reasons for this incomplete switch' observed. CHD1 may have dual roles; for example, as an activator of self-renewal genes and as repressor of pluripotency genes upon differentiation. It is, however, most likely that it is not sufficient to sacrifice chromatin plasticity for the induction of differentiation, but that other factors (not induced in the experiment) are needed for the proper definition of the path towards an appropriately differentiated chromatin structure and gene expression programme. Those nucleosome remodelling factors that serve dedicated roles as transcription co-regulators of key genes will undoubtedly be among them (see above).

A related example illustrating the importance of chromatin organization in maintaining ES cell identity comes from the work of Pannings and co-workers who studied the role of the chromatin remodelling factor p400/Tip60 in stem cell renewal (Fazzio et al., 2008). p400 is the homologue of yeast SWR1 and Drosophila Domino, remodelling ATPases involved in the exchange of histone variants. Upon removal of p400, ES cells lost their ability to form compact colonies, to associate in embryoid bodies and to generate theratomas. The differentiation capacity into derivatives of all three germ layers was defective, although the expression of some ES cell markers, such as Nanog, was maintained. The depletion of p400 affected the transcription of only $4 \%$ of the genes, but this included the activation of differentiation genes that are normally silent in ES cells. As before, the depletion of the nucleosome remodelling factor led to 
loss of the chromatin organization that characterizes the pluripotent state, but was not instructive towards a proper differentiation path.

The role of nucleosome remodellers in the maintenance of stemness seems to be conserved in evolution, as the homologue of p400 in Drosophila, Domino, was found responsible for maintenance of the somatic stem cells in fly ovaries (Xi and Xie, 2005). Interestingly, mutation of the domino gene only affected somatic stem cells, but not the neighbouring germ stem cells, whose maintenance relied on the ATPase ISWI.

\section{Concluding remarks}

In summary, it is becoming increasingly clear that some energy-consuming chromatin remodelling complexes may exert an effect as local, gene specific co-regulators of transcription, whereas others affect the epigenome on a global scale. The same ATPases may be involved in local or global actions, depending on the molecular environment defined by the associating subunits. Their effect on developmental decisions is determined through their cell type- or stage-specific expression, their targeting partners and by their ability to deal with specific chromatin substrates. These general statements should, however, not serve to cover up how little we understand-in general and in detail-about the intricate contributions of the soft skills of nucleosome remodelling factors in taking the tough decisions demanded by the faithful development of an organism.

\section{Conflict of interest}

The authors declare no conflict of interest.

\section{Acknowledgements}

This work was supported by the Deutsche Forschungsgemeinschaft through SPP1356 'Pluripotency'.

\section{References}

Allis CD, Jenuwein T, Reinberg D (2007). Epigenetics. Cold Spring Harbor Laboratory Press: New York, 502 pp.

Altaf M, Auger A, Covic M, Cote J (2009). Connection between histone $\mathrm{H} 2 \mathrm{~A}$ variants and chromatin remodeling complexes. Biochem Cell Biol 87: 35-50.

Arney KL, Fisher AG (2004). Epigenetic aspects of differentiation. J Cell Sci 117(Part 19): 4355-4363.

Azuara V, Perry P, Sauer S, Spivakov M, Jorgensen HF, John RM et al. (2006). Chromatin signatures of pluripotent cell lines. Nat Cell Biol 8: 532-538.

Badenhorst P, Voas M, Rebay I, Wu C (2002). Biological functions of the ISWI chromatin remodeling complex NURF. Genes Dev 16: 3186-3198.

Badenhorst P, Xiao H, Cherbas L, Kwon SY, Voas M, Rebay I et al. (2005). The Drosophila nucleosome remodeling factor NURF is required for ecdysteroid signaling and metamorphosis. Genes Dev 19: 2540-2545.

Bajpai R, Chen DA, Rada-Iglesias A, Zhang J, Xiong Y, Helms J et al. (2010). CHD7 cooperates with PBAF to control multipotent neural crest formation. Nature 463: 958-962.

Becker PB (2002). Nucleosome sliding: facts and fiction. EMBO J 21: 4749-4753.

Becker PB, Horz W (2002). ATP-dependent nucleosome remodeling. Annu Rev Biochem 71: 247-273.
Bernstein BE, Mikkelsen TS, Xie X, Kamal M, Huebert DJ, Cuff J et al. (2006). A bivalent chromatin structure marks key developmental genes in embryonic stem cells. Cell 125: 315-326.

Bonisch C, Nieratschker SM, Orfanos NK, Hake SB (2008). Chromatin proteomics and epigenetic regulatory circuits. Expert Rev Proteomics 5: 105-119.

Bowen NJ, Fujita N, Kajita M, Wade PA (2004). Mi-2/NuRD: multiple complexes for many purposes. Biochim Biophys Acta 1677: 52-57.

Cadigan KM (2008). Wnt-beta-catenin signaling. Curr Biol 18: R943-R947.

Cadigan KM, Liu YI (2006). Wnt signaling: complexity at the surface. J Cell Sci 119(Part 3): 395-402.

Chalkley GE, Moshkin YM, Langenberg K, Bezstarosti K, Blastyak A, Gyurkovics H et al. (2008). The transcriptional coactivator SAYP is a trithorax group signature subunit of the PBAP chromatin remodeling complex. Mol Cell Biol 28: 2920-2929.

Chioda M, Vengadasalam S, Kremmer E, Eberharter A, Becker PB (2010). Developmental role for dACF1-containing nucleosome remodelers in chromatin organisation. manuscript in preparation.

Clapier CR, Cairns BR (2009). The biology of chromatin remodeling complexes. Annu Rev Biochem 78: 273-304.

Corona DF, Siriaco G, Armstrong JA, Snarskaya N, McClymont SA, Scott MP et al. (2007). ISWI regulates higher-order chromatin structure and histone $\mathrm{H} 1$ assembly in vivo. PLoS Biol 5: e232.

Corona DF, Tamkun JW (2004). Multiple roles for ISWI in transcription, chromosome organization and DNA replication. Biochim Biophys Acta 1677: 113-119.

de la Serna IL, Ohkawa Y, Imbalzano AN (2006). Chromatin remodelling in mammalian differentiation: lessons from ATP-dependent remodellers. Nat Rev Genet 7: 461-473.

Deuring R, Fanti L, Armstrong JA, Sarte M, Papoulas O, Prestel $M$ et al. (2000). The ISWI chromatin-remodeling protein is required for gene expression and the maintenance of higher order chromatin structure in vivo. Mol Cell 5: 355-365.

Dimitrov S, Almouzni G, Dasso M, Wolffe AP (1993). Chromatin transitions during early Xenopus embryogenesis: changes in histone $\mathrm{H} 4$ acetylation and in linker histone type. Dev Biol 160: 214-227.

Fan Y, Nikitina T, Zhao J, Fleury TJ, Bhattacharyya R, Bouhassira EE et al. (2005). Histone H1 depletion in mammals alters global chromatin structure but causes specific changes in gene regulation. Cell 123: 1199-1212.

Fazzio TG, Huff JT, Panning B (2008). An RNAi screen of chromatin proteins identifies Tip60-p400 as a regulator of embryonic stem cell identity. Cell 134: 162-174.

Flaus A, Martin DM, Barton GJ, Owen-Hughes T (2006). Identification of multiple distinct Snf2 subfamilies with conserved structural motifs. Nucleic Acids Res 34: 2887-2905.

Fyodorov DV, Blower MD, Karpen GH, Kadonaga JT (2004). Acf1 confers unique activities to ACF/CHRAC and promotes the formation rather than disruption of chromatin in vivo. Genes Dev 18: 170-183.

Gao H, Lukin K, Ramirez J, Fields S, Lopez D, Hagman J (2009). Opposing effects of SWI/SNF and Mi-2/NuRD chromatin remodeling complexes on epigenetic reprogramming by EBF and Pax5. Proc Natl Acad Sci USA 106: 11258-11263.

Gaspar-Maia A, Alajem A, Polesso F, Sridharan R, Mason MJ, Heidersbach A et al. (2009). Chd1 regulates open chromatin and pluripotency of embryonic stem cells. Nature 460: 863-868.

Giadrossi S, Dvorkina M, Fisher AG (2007). Chromatin organization and differentiation in embryonic stem cell models. Curr Opin Genet Dev 17: 132-138.

Godde JS, Ura K (2009). Dynamic alterations of linker histone variants during development. Int J Dev Biol 53: 215-224.

Groth A, Rocha W, Verreault A, Almouzni G (2007). Chromatin challenges during DNA replication and repair. Cell 128: 721-733. 
Hanai K, Furuhashi H, Yamamoto T, Akasaka K, Hirose S (2008). RSF governs silent chromatin formation via histone H2Av replacement. PLoS Genet 4: e1000011.

Ho L, Jothi R, Ronan JL, Cui K, Zhao K, Crabtree GR (2009a). An embryonic stem cell chromatin remodeling complex, esBAF, is an essential component of the core pluripotency transcriptional network. Proc Natl Acad Sci USA 106: 5187-5191.

Ho L, Ronan JL, Wu J, Staahl BT, Chen L, Kuo A et al. (2009b). An embryonic stem cell chromatin remodeling complex, esBAF, is essential for embryonic stem cell self-renewal and pluripotency. Proc Natl Acad Sci USA 106: 5181-5186.

Ito T, Bulger M, Pazin MJ, Kobayashi R, Kadonaga JT (1997). $\mathrm{ACF}$, an ISWI-containing and ATP-utilizing chromatin assembly and remodeling factor. Cell 90: 145-155.

Ito T, Levenstein ME, Fyodorov DV, Kutach AK, Kobayashi R, Kadonaga JT (1999). ACF consists of two subunits, Acf1 and ISWI, that function cooperatively in the ATP-dependent catalysis of chromatin assembly. Genes Dev 13: 1529-1539.

Izzo A, Kamieniarz K, Schneider R (2008). The histone H1 family: specific members, specific functions? Biol Chem 389: 333-343.

Kaji K, Caballero IM, MacLeod R, Nichols J, Wilson VA, Hendrich B (2006). The NuRD component Mbd3 is required for pluripotency of embryonic stem cells. Nat Cell Biol 8: 285-292.

Keenen B, de la Serna IL (2009). Chromatin remodeling in embryonic stem cells: regulating the balance between pluripotency and differentiation. J Cell Physiol 219: 1-7.

Kia SK, Gorski MM, Giannakopoulos S, Verrijzer CP (2008). SWI/SNF mediates polycomb eviction and epigenetic reprogramming of the INK4b-ARF-INK4a locus. Mol Cell Biol 28 3457-3464.

Konev AY, Tribus M, Park SY, Podhraski V, Lim CY, Emelyanov AV et al. (2007). CHD1 motor protein is required for deposition of histone variant H3.3 into chromatin in vivo. Science 317: 1087-1090.

Kusch T, Workman JL (2007). Histone variants and complexes involved in their exchange. Subcell Biochem 41: 91-109.

Kwon CS, Wagner D (2007). Unwinding chromatin for development and growth: a few genes at a time. Trends Genet 23: 403-412.

Kwon SY, Xiao H, Glover BP, Tjian R, Wu C, Badenhorst P (2008). The nucleosome remodeling factor (NURF) regulates genes involved in Drosophila innate immunity. Dev Biol 316 538-547.

Kwon SY, Xiao H, Wu C, Badenhorst P (2009). Alternative splicing of NURF301 generates distinct NURF chromatin remodeling complexes with altered modified histone binding specificities. PLoS Genet 5: e1000574.

Langst G, Becker PB (2004). Nucleosome remodeling: one mechanism, many phenomena? Biochim Biophys Acta 1677: 58-63.

Linder B, Mentele E, Mansperger K, Straub T, Kremmer E, Rupp RA (2007). CHD4/Mi-2beta activity is required for the positioning of the mesoderm/neuroectoderm boundary in Xenopus. Genes Dev 21: 973-983.

Liu YI, Chang MV, Li HE, Barolo S, Chang JL, Blauwkamp TA et al. (2008). The chromatin remodelers ISWI and ACF1 directly repress Wingless transcriptional targets. Dev Biol 323: $41-52$.

Logan CY, Nusse R (2004). The Wnt signaling pathway in development and disease. Annu Rev Cell Dev Biol 20: 781-810.

Lusser A, Urwin DL, Kadonaga JT (2005). Distinct activities of CHD1 and ACF in ATP-dependent chromatin assembly. Nat Struct Mol Biol 12: 160-166.

Maier VK, Chioda M, Becker PB (2008a). ATP-dependent chromatosome remodeling. Biol Chem 389: 345-352.

Maier VK, Chioda M, Rhodes D, Becker PB (2008b). ACF catalyses chromatosome movements in chromatin fibres. EMBO J 27: 817-826.
Meshorer E, Misteli T (2006). Chromatin in pluripotent embryonic stem cells and differentiation. Nat Rev Mol Cell Biol 7: 540-546.

Meshorer E, Yellajoshula D, George E, Scambler PJ, Brown DT, Misteli T (2006). Hyperdynamic plasticity of chromatin proteins in pluripotent embryonic stem cells. Dev Cell 10: 105-116.

Mito Y, Henikoff JG, Henikoff S (2007). Histone replacement marks the boundaries of cis-regulatory domains. Science 315: 1408-1411.

Ner SS, Blank T, Perez-Paralle ML, Grigliatti TA, Becker PB, Travers AA (2001). HMG-D and histone H1 interplay during chromatin assembly and early embryogenesis. J Biol Chem 276: 37569-37576.

Ner SS, Travers AA (1994). HMG-D, the Drosophila melanogaster homologue of HMG 1 protein, is associated with early embryonic chromatin in the absence of histone $\mathrm{H} 1$. EMBO J 13: $1817-1822$

Nusse R, Fuerer C, Ching W, Harnish K, Logan C, Zeng A et al. (2008). Wnt signaling and stem cell control. Cold Spring Harb Symp Quant Biol 73: 59-66.

Orsi GA, Couble P, Loppin B (2009). Epigenetic and replacement roles of histone variant $\mathrm{H} 3.3$ in reproduction and development. Int J Dev Biol 53: 231-243.

Pietersen AM, van Lohuizen M (2008). Stem cell regulation by polycomb repressors: postponing commitment. Curr Opin Cell Biol 20: 201-207.

Rodriguez-Paredes M, Ceballos-Chavez M, Esteller M, GarciaDominguez M, Reyes JC (2009). The chromatin remodeling factor CHD8 interacts with elongating RNA polymerase II and controls expression of the cyclin E2 gene. Nucleic Acids Res 37: 2449-2460.

Routh A, Sandin S, Rhodes D (2008). Nucleosome repeat length and linker histone stoichiometry determine chromatin fiber structure. Proc Natl Acad Sci USA 105: 8872-8877.

Siriaco G, Deuring R, Chioda M, Becker PB, Tamkun JW (2009). Drosophila ISWI regulates the association of histone $\mathrm{H} 1$ with interphase chromosomes in vivo. Genetics 182: 661-669.

Song H, Spichiger-Haeusermann C, Basler K (2009). The ISWIcontaining NURF complex regulates the output of the canonical Wingless pathway. EMBO Rep 10: 1140-1146.

Srinivasan S, Dorighi KM, Tamkun JW (2008). Drosophila Kismet regulates histone $\mathrm{H} 3$ lysine 27 methylation and early elongation by RNA polymerase II. PLoS Genet 4: e1000217.

Suganuma T, Workman JL (2008). Crosstalk among histone modifications. Cell 135: 604-607.

Svotelis A, Gevry N, Gaudreau L (2009). Regulation of gene expression and cellular proliferation by histone H2A.Z. Biochem Cell Biol 87: 179-188.

Swaminathan J, Baxter EM, Corces VG (2005). The role of histone $\mathrm{H} 2 \mathrm{Av}$ variant replacement and histone $\mathrm{H} 4$ acetylation in the establishment of Drosophila heterochromatin. Genes Dev 19: 65-76.

Terriente J, Perea D, Suzanne M, Diaz-Benjumea FJ (2008). The Drosophila gene $\mathrm{zfh} 2$ is required to establish proximal-distal domains in the wing disc. Dev Biol 320: 102-112.

Thompson BA, Tremblay V, Lin G, Bochar DA (2008). CHD8 is an ATP-dependent chromatin remodeling factor that regulates beta-catenin target genes. Mol Cell Biol 28: 3894-3904.

Tsukiyama T, Daniel C, Tamkun J, Wu C (1995). ISWI, a member of the SWI2/SNF2 ATPase family, encodes the $140 \mathrm{kDa}$ subunit of the nucleosome remodeling factor. Cell 83: 1021-1026.

Varga-Weisz PD, Becker PB (2006). Regulation of higher-order chromatin structures by nucleosome-remodelling factors. Curr Opin Genet Dev 16: 151-156.

Varga-Weisz PD, Wilm M, Bonte E, Dumas K, Mann M, Becker PB (1997). Chromatin-remodelling factor CHRAC contains the ATPases ISWI and topoisomerase II. Nature 388: 598-602.

Wood C, Snijders A, Williamson J, Reynolds C, Baldwin J, Dickman M (2009). Post-translational modifications of the 
linker histone variants and their association with cell mechanisms. FEBS J 276: 3685-3697.

Woodcock CL, Skoultchi AI, Fan Y (2006). Role of linker histone in chromatin structure and function: H1 stoichiometry and nucleosome repeat length. Chromosome Res 14: 17-25.

Xi R, Xie T (2005). Stem cell self-renewal controlled by chromatin remodeling factors. Science 310: 1487-1489.
Yoo AS, Crabtree GR (2009). ATP-dependent chromatin remodeling in neural development. Curr Opin Neurobiol 19: 120-126.

Yoshida T, Hazan I, Zhang J, Ng SY, Naito T, Snippert HJ et al. (2008). The role of the chromatin remodeler Mi-2beta in hematopoietic stem cell self-renewal and multilineage differentiation. Genes Dev 22: 1174-1189. 